\title{
Fuzzy Stability and Synchronization of New 3D Chaotic Systems
}

\author{
Masoud Taleb Ziabari \\ Faculty of Engineering, Computer Engineering Group, Mehr Aeen University, Bandar Anzali, Iran \\ Email: m.t.ziabari@gmail.com \\ Ali Moarefianpur \\ Faculty of Engineering, Islamic Azad University Science and Research Branch, Tehran, Iran \\ Email: moarefian@srbiau.ac.ir \\ Marjan Morvarid \\ Department of Computer Engineering, Ahrar Institute of Technology and Higher Education, Rasht, Iran \\ Email: ma.morvarid@gmail.com
}

\begin{abstract}
This paper presents fuzzy model-based designs for control and synchronization of new chaotic system. The T-S fuzzy models for new chaotic systems are exactly derived. Then the asymptotic stability and synchronization are achieved by generalized backstepping method. On the other hand, this paper presents fuzzy model-based designs for synchronization of another chaotic system. Based on the T-S fuzzy new chaotic models, the fuzzy controllers for two different chaotic synchronization are designed via the active control technique. Numerical simulation results are presented to show the effectiveness of the proposed method.
\end{abstract}

Index Terms-T-S Fuzzy Model, Generalized Backstepping Method, Active Control, Stability, Synchronization.

\section{INTRODUCTION}

Control of complex irregular dynamics has evolved as one of the central issues in applied nonlinear science during the last decade. Nowadays the notion of chaos control involving stabilization of unstable periodic or stationary states in nonlinear dynamic systems has been extended to a much wider class of problems. Since the discovery of chaos synchronization introduced in [1], there have been tremendous interests in studying the synchronization of chaotic systems. Recently, much research on the fuzzy model-based designs to stability and synchronization for chaotic systems have been carried out based on Takagi-Sugeno (T-S) fuzzy models [2-4]. In [5], a fuzzy model-based designs for Chen's chaotic stability and synchronization have been proposed. Based on the fuzzy hyperchaotic models, simpler fuzzy controllers have been designed for synchronizing hyperchaotic systems in [6]. In [7], a novel IMRAFC for a class of nonlinear chaotic systems subject to disturbances was proposed. A robust model reference fuzzy controller and the adaptation laws for the identification system were derived using an appropriate Lyapunov function. [8] have considered the problem of synchronizing a class of uncertain nonlinear systems by using adaptive fuzzy sliding mode control technique. In [9], authors have considered the robust stabilization and synchronization problems for nonlinear energy resource systems based on T-S fuzzy model. By designing the fuzzy state-feedback controllers, several sufficient conditions have been obtained to ensure the stability, robust stability and synchronization of nonlinear energy resources systems, respectively. A new adaptive interval type-2 fuzzy neural network (FNN) control for chaos synchronization between two different chaotic systems was proposed to handle the training data corrupted by noise or rule uncertainties involving external disturbances [10]. By investigating synchronization of chaotic systems, the structure of drive-response lag synchronization for fuzzy chaos system based on fuzzy observer was proposed in [11]. A Genetic based Fuzzy PID controller [12] has been proposed to synchronization task of chaotic systems in which one system has been considered as "master" whilst the other system has been treated as "slave" (a perturbed system with uncertainty and disturbance). In [13], two identical generalized Lorenz systems have been synchronized by a fuzzy controller based on mamdani approach and stability of the proposed scheme has been established by the Lyapunov stability theorem. In [14], the new 3 dimensional chaotic system was first investigated and then utilized an intelligent controller based on brain emotional learning (BELBIC), this new chaotic system was synchronized. In [15], stabilization and synchronization problem of the new hyperchaotic system was investigated. state dependent Riccati equation (SDRE) was applied to new hyperchaotic system in three ways. Stabilized system with know parameters, stabilized system with unknow parameters, sysnchronized system.

The rest of the paper is organized as follows. In Section 2, the T-S fuzzy models will be presented for a 
new 3D chaotic system. In Section 3, fuzzy stability of chaotic system is presented. In Section 4, synchronization between two new fuzzy chaotic system is achieved by generalized backstepping method is described. In Section 5 , synchronization between two different fuzzy chaotic systems is achieved by active control. Finally, Section 6 provides, conclusion of this work.

\section{T-S FUZZY MODEL OF NEW 3D CHAOTIC SYSTEM}

The T-S fuzzy dynamic model originates from Takagi and Sugeno [16], which is described by fuzzy IF-THEN rules where the consequent parts represent local linear models. Consider a continuous-time nonlinear dynamic equation as follows:

$$
\dot{x}(t)=f(x(t))+g(x(t)) u(t)
$$

Then the T-S fuzzy model is composed of the following rules:

Rule i: if $z_{1}(t)$ is $M_{i 1} \& \cdots \& z_{p}(t)$ is $M_{i p}$ then $\dot{x}(t)=$ $A_{i} x(t)+B u(t), \quad i=1,2, \cdots, r$.

Where $Z_{1}(t) \sim Z_{p}(t)$ are the premise variableswhich would consist of the states of the system, $M_{i j}(j=$ $1,2, \ldots, p$ ) are fuzzy sets, $r$ is the number of fuzzy rules, $A_{i}, B$ are system matrices with appropriate dimensions. The final output of the fuzzy system is inferred as follows:

$$
\dot{x}(t)=\sum_{i=1}^{r} h_{i}(z(t))\left(A_{i} x(t)+B u(t)\right)
$$

Where,

$$
h_{i}(z(t))=\frac{w_{i}(z(t))}{\sum_{i=1}^{r} w_{i}(z(t))}, w_{i}(z(t))=\prod_{j=1}^{p} M_{i j}\left(z_{j}(t)\right)
$$

Lemma [17]. The equilibrium of the continuous fuzzy system (3) with $u(t)=0$ is globally asymptotically stable if there exists a common positive definite matrix $P$ such that

$$
A_{i}^{T} P+P A_{i}<0, \quad i=1,2, \cdots, r
$$

that is, a common $P$ has to exist for all subsystems.

Recently, Sara Dadras and Hamid Reza Momeni constructed the 3D autonomous chaotic system [18]. The system is described by.

$$
\begin{aligned}
& \dot{x}=y-a x+b y z \\
& \dot{y}=c y-x z+z \\
& \dot{z}=d x y-h z
\end{aligned}
$$

Where $a, b, c, d, h$ are positive constants and $x, y, z$ are variables of the system, when $a=3, b=2.7, c=$ $4.7, d=2, h=9$, the system (6) is chaotic. See Figure 1 .
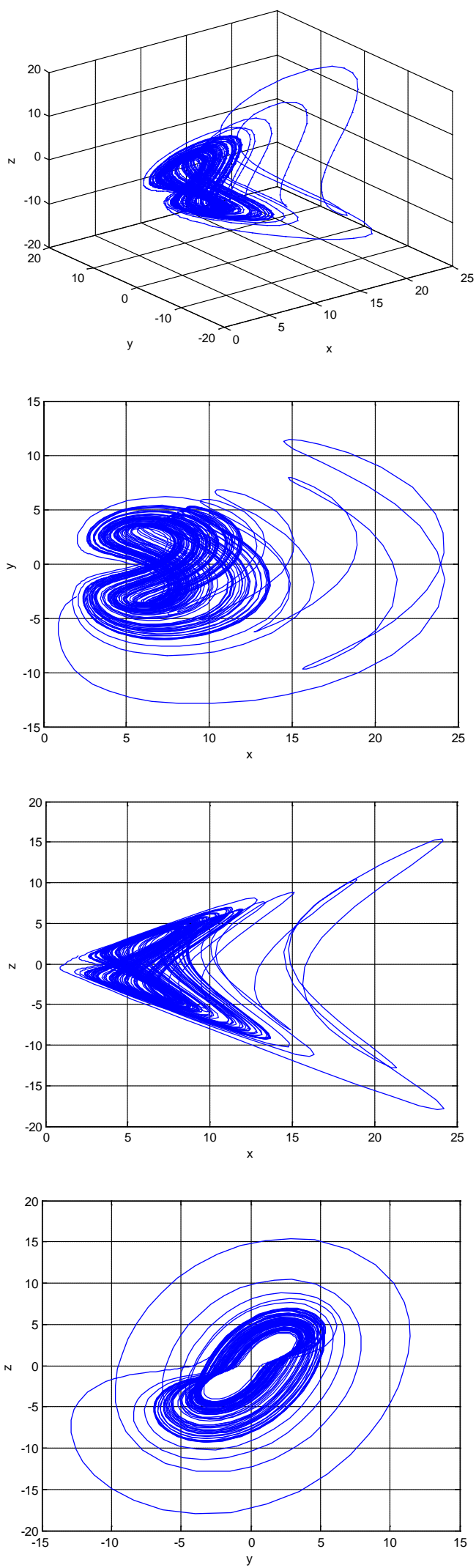

Fig 1. Strange attractors system (6)

Assume that $x \in[-g, g], y \in[-g, g]$ and $g>0$, then system (6) can be exactly represented by T-S fuzzy model as following: 
Rule 1: if $x$ is $M_{1}$ \& $y$ is $N_{1}$ then $\dot{X}(t)=A_{1} X(t)$

Rule 2: if $x$ is $M_{1}$ \& $y$ is $N_{2}$ then $\dot{X}(t)=A_{2} X(t)$

Rule 3: if $x$ is $M_{2}$ \& $y$ is $N_{1}$ then $\dot{X}(t)=A_{3} X(t)$

Rule 4: if $x$ is $M_{2}$ \& $y$ is $N_{2}$ then $\dot{X}(t)=A_{4} X(t)$

Here,

$$
\begin{gathered}
X=\left[\begin{array}{lll}
x & y & z
\end{array}\right]^{T} \\
M_{1}(x)=\frac{1}{2}\left(1+\frac{x}{g}\right), M_{2}(x)=\frac{1}{2}\left(1-\frac{x}{g}\right) \\
N_{1}(y)=\frac{1}{2}\left(1+\frac{y}{g}\right), N_{2}(y)=\frac{1}{2}\left(1-\frac{y}{g}\right)
\end{gathered}
$$

And states matrixes are:

$$
\begin{aligned}
A_{1} & =\left[\begin{array}{ccc}
-a & 1 & b g \\
0 & c & 1-g \\
0 & d g & -h
\end{array}\right] \\
A_{2} & =\left[\begin{array}{ccc}
-a & 1 & -b g \\
0 & c & 1-g \\
0 & d g & -h
\end{array}\right] \\
A_{3} & =\left[\begin{array}{ccc}
-a & 1 & b g \\
0 & c & 1+g \\
0 & -d g & -h
\end{array}\right] \\
A_{4} & =\left[\begin{array}{ccc}
-a & 1 & -b g \\
0 & c & 1+g \\
0 & -d g & -h
\end{array}\right]
\end{aligned}
$$

Rule 1: if $x$ is $M_{1}$ \& $y$ is $N_{1}$ then $\dot{X}(t)=A_{1} X(t)+$ $B u(t)$

Rule 2: if $x$ is $M_{1}$ \& $y$ is $N_{2}$ then $\dot{X}(t)=A_{2} X(t)+$ $B u(t)$

Rule 3: if $x$ is $M_{2}$ \& $y$ is $N_{1}$ then $\dot{X}(t)=A_{3} X(t)+$ $B u(t)$

Rule 4: if $x$ is $M_{2}$ \& $y$ is $N_{2}$ then $\dot{X}(t)=A_{4} X(t)+$ $B u(t)$

Where $B=\left[\begin{array}{l}0 \\ 1 \\ 0\end{array}\right]$. According to the Generalized Backstepping Method [19, 20] theorem, the control signal will be obtained:

Rule 1: if $x$ is $M_{1}$ \& $y$ is $N_{1}$ then

$u=-\left[\begin{array}{lll}1-a b g & 1+c+b g & 1-g+d g+b g+(b g)^{2}\end{array}\right] X(t)$

Rule 2: if $x$ is $M_{1}$ \& $y$ is $N_{2}$ then

$u=-\left[\begin{array}{lll}1+a b g & 1+c-b g & 1-g+d g-b g+(b g)^{2}\end{array}\right] X(t)$

Rule 3: if $x$ is $M_{2}$ \& $y$ is $N_{1}$ then

$u=-\left[\begin{array}{lll}1-a b g & 1+c+b g & 1+g-d g+b g+(b g)^{2}\end{array}\right] X(t)$

Rule 4: if $x$ is $M_{2}$ \& $y$ is $N_{2}$ then

$u=-\left[\begin{array}{lll}1+a b g & 1+c-b g & 1+g-d g-b g+(b g)^{2}\end{array}\right] X(t)$

Figure 3 shows that $(x, y, z)$ states of new fuzzy chaotic system (7) can be stabilized with the control laws $u(12)$ to the origin point $(0,0,0)$. The time response of the control input $u$ for the stabilization new fuzzy chaotic system (7) shown in Figure 4.
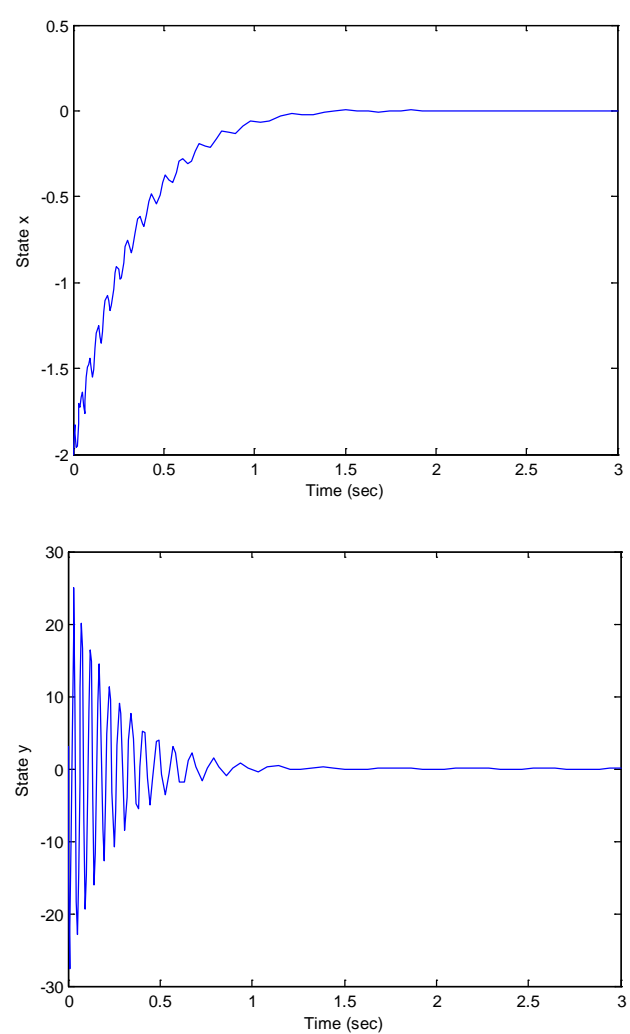

For new chaotic fuzzy system (7), the following fuzzy controller is used to stabilize it.

To support our analysis to be carried out in the following sections, some existing results are needed.

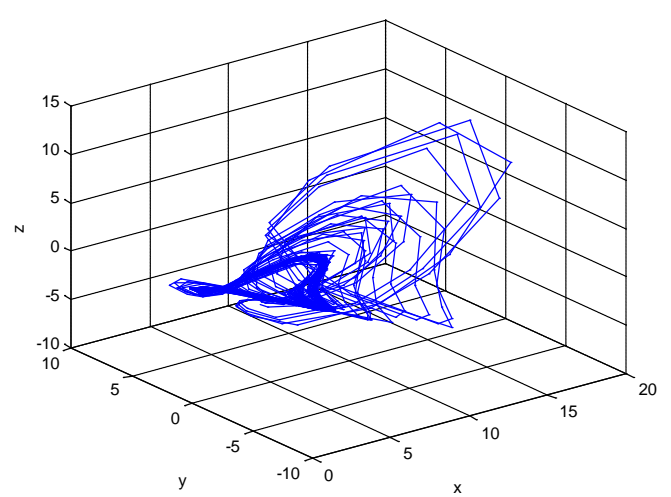

Fig 2. Chaotic behavior of fuzzy system (7).

\section{FUZZY STABILITY OF NEW CHAOTIC SYSTEM}




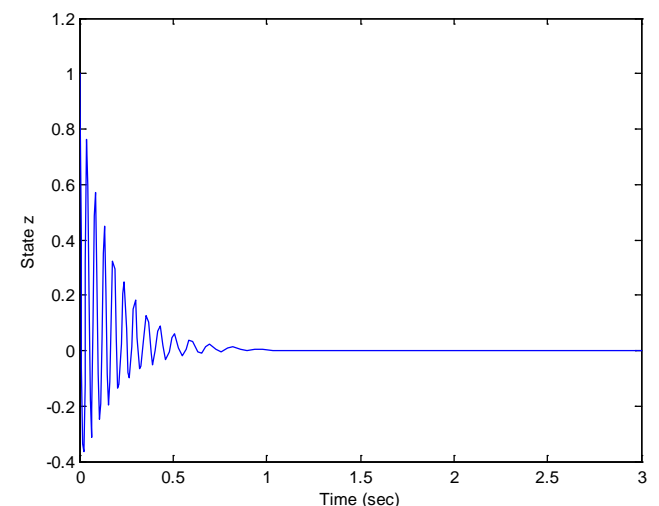

Fig 3 . The time response of the states $(x, y, z)$ for the controlled new fuzzy chaotic system (7) where the control inputs is (12).

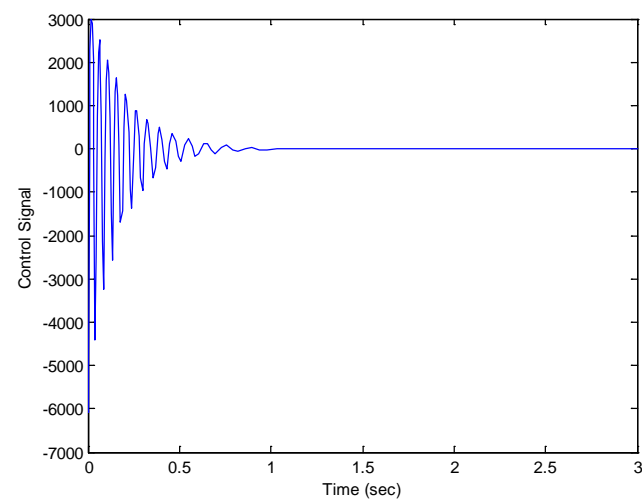

Fig 4. The time response of the control input .

Substituting equation (12) into equation (11) yields.

Rule 1: if $x$ is $M_{1}$ \& $y$ is $N_{1}$ then $\dot{X}(t)=A_{1} X(t)$

Rule 2: if $x$ is $M_{1}$ \& $y$ is $N_{2}$ then $\dot{X}(t)=A_{2} X(t)$

Rule 3: if $x$ is $M_{2}$ \& $y$ is $N_{1}$ then $\dot{X}(t)=A_{3} X(t)$

Rule 4: if $x$ is $M_{2}$ \& $y$ is $N_{2}$ then $\dot{X}(t)=A_{4} X(t)$

Where

$A_{1}=\left[\begin{array}{ccc}-a & 1 & b g \\ a b g-1 & -b g-1 & -d g-b g-(b g)^{2} \\ 0 & d g & -h\end{array}\right]$
$A_{2}=\left[\begin{array}{ccc}-a & 1 & -b g \\ -a b g-1 & b g-1 & -d g+b g-(b g)^{2} \\ 0 & d g & -h\end{array}\right]$
$A_{3}=\left[\begin{array}{ccc}-a & 1 & b g \\ a b g-1 & -b g-1 & d g-b g-(b g)^{2} \\ 0 & -d g & -h \\ -a & 1 & -b g \\ -a b g-1 & b g-1 & d g+b g-(b g)^{2} \\ 0 & -d g & -h\end{array}\right]$

We select $Q=I_{3 \times 3}$ and the matrix $P$ will be obtained:

$$
A_{i}^{T} P+P A_{i}=-Q, i=1,2,3,4
$$

$$
P=\left[\begin{array}{ccc}
0.1429 & -0.714 & 61.9693 \\
-0.714 & 0.2143 & -274.9887 \\
61.9693 & -274.9887 & 200466.879
\end{array}\right]
$$

\section{FuZZy SynChronization Of NEW CHAOtiC System}

In order to achieve the behavior of the synchronization between two new fuzzy chaotic system by using the generalized backstepping metod, suppose the drive system (7) and the slave system takes the following from.

Rule 1: if $\tilde{x}$ is $M_{1}$ \& $\tilde{y}$ is $N_{1}$ then $\dot{\tilde{X}}(t)=A_{1} \tilde{X}(t)+$ $B u(t)$

Rule 2: if $\tilde{x}$ is $M_{1}$ \& $\tilde{y}$ is $N_{2}$ then $\dot{\tilde{X}}(t)=A_{2} \tilde{X}(t)+$ $B u(t)$

Rule 3: if $\tilde{x}$ is $M_{2}$ \& $\tilde{y}$ is $N_{1}$ then $\dot{\tilde{X}}(t)=A_{3} \tilde{X}(t)+$ $B u(t)$

Rule 4: if $\tilde{x}$ is $M_{2}$ \& $\tilde{y}$ is $N_{2}$ then $\dot{\tilde{X}}(t)=A_{4} \tilde{X}(t)+$ $B u(t)$

Where

$$
\begin{gathered}
\tilde{X}=\left[\begin{array}{lll}
\tilde{x} & \tilde{y} & \tilde{z}
\end{array}\right]^{T}, \\
u(t)=\left[\begin{array}{ll}
u_{1}(t) & u_{2}(t)
\end{array}\right]^{T}, \\
B=\left[\begin{array}{ll}
0 & 0 \\
1 & 0 \\
0 & 1
\end{array}\right]
\end{gathered}
$$

Then the defuzzification process is given as

$$
\dot{\tilde{X}}(t)=\sum_{i=1}^{4} M_{i}(\tilde{x}) N_{i}(\tilde{y})\left(A_{i} \tilde{X}(t)+B u(t)\right)
$$

Assume that $e(t)=\tilde{X}(t)-X(t)$, we obtain $\dot{e}(t)=$

$\sum_{i=1}^{4} M_{i}(x) N_{i}(y) A_{i} X(t)-\sum_{i=1}^{4} M_{i}(\tilde{x}) N_{i}(\tilde{y})\left(A_{i} \tilde{X}(t)+\right.$ $B u(t))$

According to the Generalized Backstepping Method theorem, the control signals will be obtained:

Rule 1: if $\tilde{x}$ is $M_{1}$ \& $\tilde{y}$ is $N_{1}$ then $\left[\begin{array}{l}u_{1}(t) \\ u_{2}(t)\end{array}\right]=$ $\left[\begin{array}{ccc}a-2 & -(c+3) & -(b g-g+1) \\ -(b g+1) & -d g & h-2\end{array}\right] e(t)$

Rule 2: if $\tilde{x}$ is $M_{1}$ \& $\tilde{y}$ is $N_{2}$ then $\left[\begin{array}{l}u_{1}(t) \\ u_{2}(t)\end{array}\right]=$ $\left[\begin{array}{ccc}a-2 & -(c+3) & b g+g-1 \\ b g-1 & -d g & h-2\end{array}\right] e(t)$

Rule 3: if $\tilde{x}$ is $M_{2}$ \& $\tilde{y}$ is $N_{1}$ then $\left[\begin{array}{l}u_{1}(t) \\ u_{2}(t)\end{array}\right]=$ $\left[\begin{array}{ccc}a-2 & -(c+3) & -(b g+g+1) \\ -(b g+1) & d g & h-2\end{array}\right] e(t)$ 
Rule 4: if $\tilde{x}$ is $M_{2}$ \& $\tilde{y}$ is $N_{2}$ then $\left[\begin{array}{l}u_{1}(t) \\ u_{2}(t)\end{array}\right]=$

$\left[\begin{array}{ccc}a-2 & -(c+3) & b g-g-1 \\ b g-1 & d g & h-2\end{array}\right] e(t)$

The initial values of the the drive and response systems are $x_{1}(0)=-2, y_{1}(0)=1, z_{1}(0)=-3$ and $x_{2}(0)=$ $1, y_{2}(0)=-1, z_{2}(0)=1$ respectively. The time response of $x, y, z$ states for drive system (7) and the response system (17) shown in Figure 5. Synchronization errors $\left(e_{x}, e_{y}, e_{z}\right)$ in the new fuzzy chaotic systems shown in Figure 6. The time response of the control inputs $\left(u_{1}, u_{2}\right)$ for the synchronization new fuzzy chaotic systems shown in Figure 7.
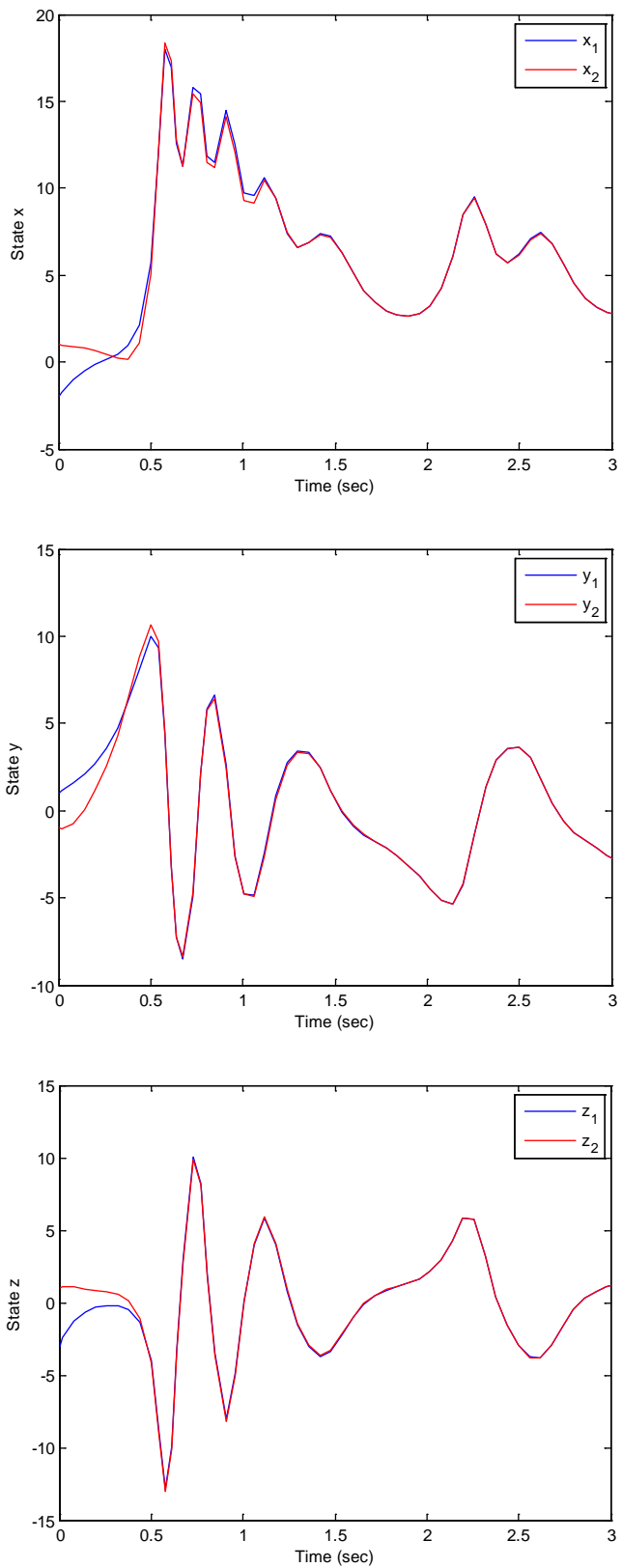

Fig 5. The time response of signals $(x, y, z)$ for drive system (7) and response system (17).
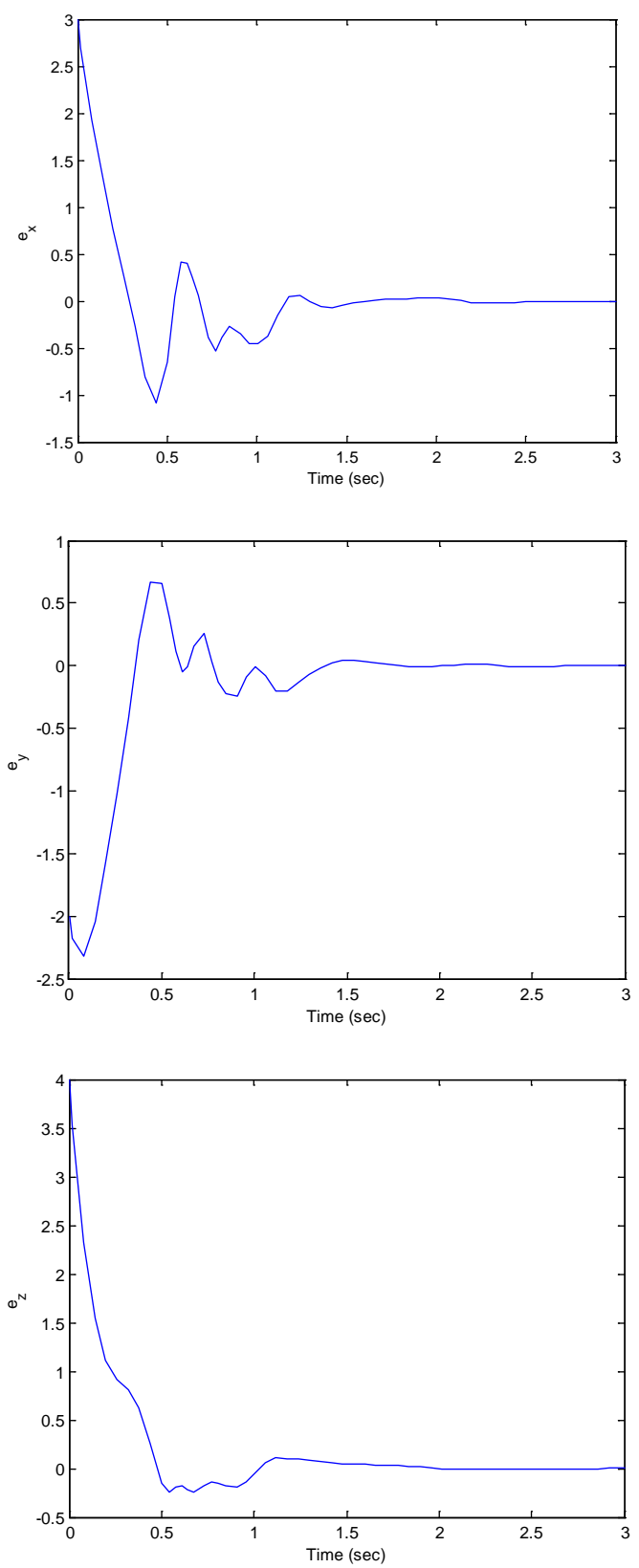

Fig 6. Synchronization errors $\left(e_{x}, e_{y}, e_{z}\right)$ in drive system (7) and response system (17).

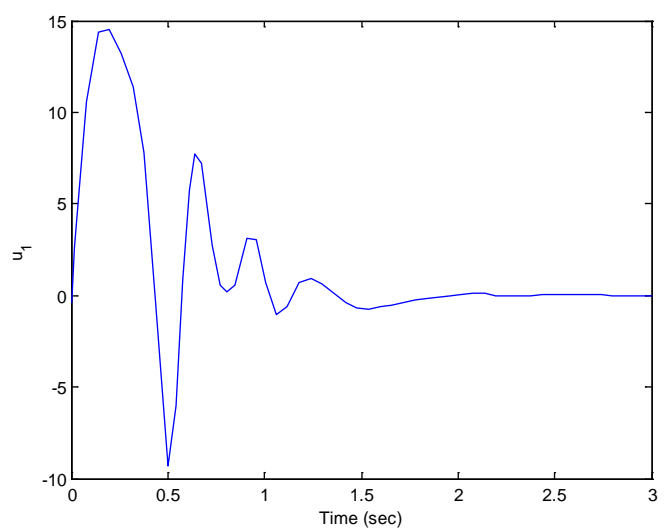




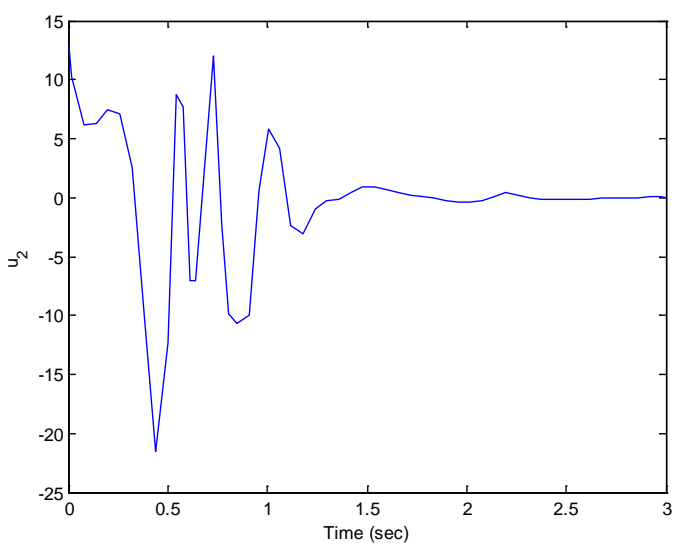

Fig 7 . The time response of the control inputs $\left(u_{1}, u_{2}\right)$ for drive system (7) and response system (17).

Substituting equation (21) into equation (17) yields.

Rule 1: if $\tilde{x}$ is $M_{1}$ \& $\tilde{y}$ is $N_{1}$ then $\dot{e}(t)=A_{1} e(t)$ Rule 2: if $\tilde{x}$ is $M_{1}$ \& $\tilde{y}$ is $N_{2}$ then $\dot{e}(t)=A_{2} e(t)$ Rule 3: if $\tilde{x}$ is $M_{2}$ \& $\tilde{y}$ is $N_{1}$ then $\dot{e}(t)=A_{3} e(t)$ Rule 4: if $\tilde{x}$ is $M_{2}$ \& $\tilde{y}$ is $N_{2}$ then $\dot{e}(t)=A_{4} e(t)$

Where

$$
\begin{aligned}
& A_{1}=A_{3}= {\left[\begin{array}{ccc}
-a & 1 & b g \\
a-2 & -3 & -b g \\
-(b g+1) & 0 & -2
\end{array}\right] } \\
& A_{2}=A_{4}=\left[\begin{array}{ccc}
-a & 1 & -b g \\
a-2 & -3 & b g \\
b g-1 & 0 & -2
\end{array}\right]
\end{aligned}
$$

We select $Q=I_{3 \times 3}$ and the matrix $P$ will be obtained:

$$
\begin{gathered}
A_{i}^{T} P+P A_{i}=-Q, i=1,2,3,4 \\
P=\left[\begin{array}{lll}
\frac{119}{576} & \frac{13}{192} & \frac{-5}{96} \\
\frac{13}{192} & \frac{109}{576} & \frac{-1}{96} \\
\frac{-5}{96} & \frac{-1}{96} & \frac{1}{4}
\end{array}\right]
\end{gathered}
$$

\section{FUZZY SyNCHRONIZATION OF TwO DIFFERENT CHAOTIC SYSTEM}

Recently, Chunlai Li, Hongmin Li and Yaonan Tong constructed the 3D autonomous chaotic system [21]. The system is described by.

$$
\begin{aligned}
\dot{x} & =-a x+b y z \\
\dot{y} & =c y-f x z \\
\dot{z} & =d y^{2}-h z
\end{aligned}
$$

Where $a, b, c, d, f, h$ are positive constants and $x, y, z$ are variables of the system, when $a=16, b=0.5, c=$ $10, d=18, f=6, h=5$, the system (26) is chaotic. See Figure 8 .
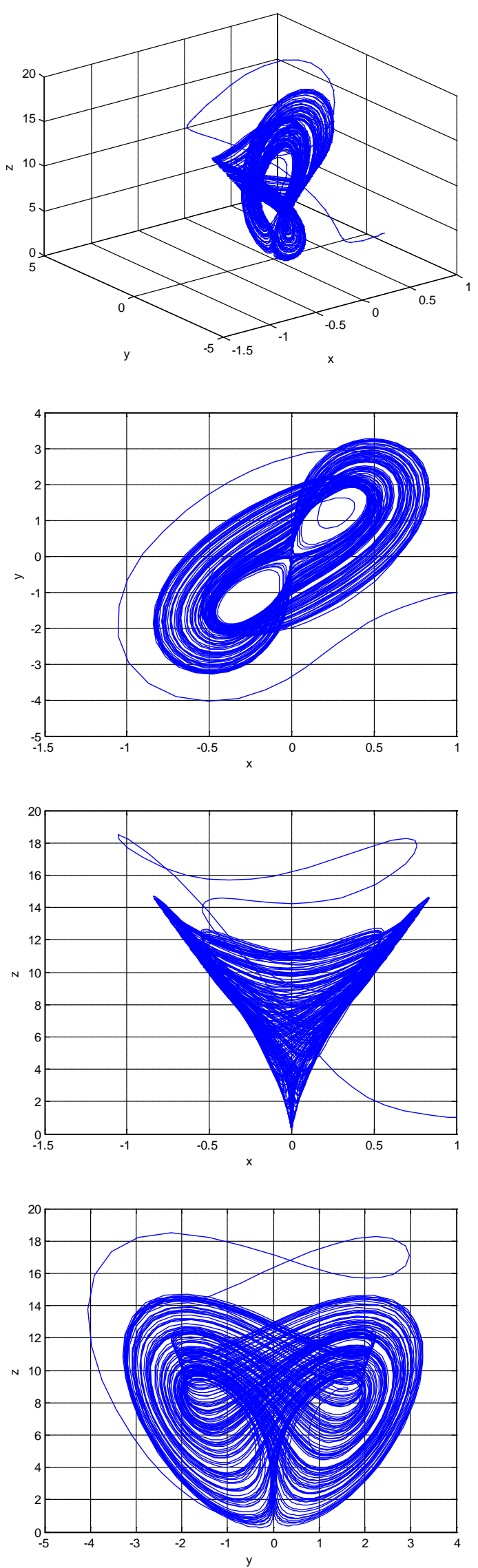

Fig 8. Strange attractors system (26) 
Assume that $x \in[-g, g], y \in[-g, g]$ and $g>0$, then system (26) can be exactly represented by T-S fuzzy model as following:

Rule 1: if $x$ is $M_{1}$ \& $y$ is $N_{1}$ then $\dot{X}(t)=A_{1} X(t)$

Rule 2: if $x$ is $M_{1}$ \& $y$ is $N_{2}$ then $\dot{X}(t)=A_{2} X(t)$

Rule 3: if $x$ is $M_{2}$ \& $y$ is $N_{1}$ then $\dot{X}(t)=A_{3} X(t)$

Rule 4: if $x$ is $M_{2}$ \& $y$ is $N_{2}$ then $\dot{X}(t)=A_{4} X(t)$

Here,

$$
\begin{gathered}
X=\left[\begin{array}{lll}
x & y & z
\end{array}\right]^{T} \\
M_{1}(x)=\frac{1}{2}\left(1+\frac{x}{g}\right), M_{2}(x)=\frac{1}{2}\left(1-\frac{x}{g}\right) \\
N_{1}(y)=\frac{1}{2}\left(1+\frac{y}{g}\right), N_{2}(y)=\frac{1}{2}\left(1-\frac{y}{g}\right)
\end{gathered}
$$

And states matrixes are:

$$
\begin{aligned}
A_{1} & =\left[\begin{array}{ccc}
-a & 0 & b g \\
0 & c & -f g \\
0 & d g & -h
\end{array}\right] \\
A_{2} & =\left[\begin{array}{ccc}
-a & 0 & -b g \\
0 & c & -f g \\
0 & -d g & -h
\end{array}\right] \\
A_{3} & =\left[\begin{array}{ccc}
-a & 0 & b g \\
0 & c & f g \\
0 & d g & -h
\end{array}\right] \\
A_{4} & =\left[\begin{array}{ccc}
-a & 0 & -b g \\
0 & c & f g \\
0 & -d g & -h
\end{array}\right]
\end{aligned}
$$

That $g=30$. So the final output of the fuzzy system (27) is inferred as follows and the chaotic behavior is shown in Figure 9.

$$
\dot{X}(t)=\sum_{i=1}^{4} M_{i}(x) N_{i}(y) A_{i} X(t)
$$

To support our analysis to be carried out in the following sections, some existing results are needed.

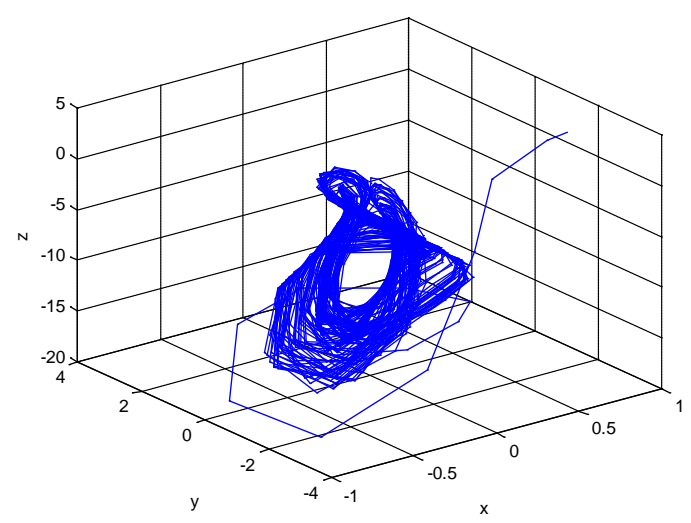

Fig 9. Chaotic behavior of fuzzy system (27).

Suppose the master and slave systems takes the following from
Master System:

Rule 1: if $x$ is $M_{1}$ \& $y$ is $N_{1}$ then $\dot{X}(t)=$ $\left[\begin{array}{ccc}-a_{1} & 0 & b_{1} g \\ 0 & c_{1} & -f_{1} g \\ 0 & d_{1} g & -h_{1}\end{array}\right] X(t)$

Rule 2: if $x$ is $M_{1}$ \& $y$ is $N_{2}$ then $\dot{X}(t)=$ $\left[\begin{array}{ccc}-a_{1} & 0 & -b_{1} g \\ 0 & c_{1} & -f_{1} g \\ 0 & -d_{1} g & -h_{1}\end{array}\right] X(t)$

Rule 3: if $x$ is $M_{2}$ \& $y$ is $N_{1}$ then $\dot{X}(t)=$ $\left[\begin{array}{ccc}-a_{1} & 0 & b_{1} g \\ 0 & c_{1} & f_{1} g \\ 0 & d_{1} g & -h_{1}\end{array}\right] X(t)$

Rule 4: if $x$ is $M_{2}$ \& $y$ is $N_{2}$ then $\dot{X}(t)=$ $\left[\begin{array}{ccc}-a_{1} & 0 & -b_{1} g \\ 0 & c_{1} & f_{1} g \\ 0 & -d_{1} g & -h_{1}\end{array}\right] X(t)$

And

\section{Slave System:}

Rule 1: if $\tilde{x}$ is $M_{1}$ \& $\tilde{y}$ is $N_{1}$ then $\dot{\tilde{X}}(t)=$ $\left[\begin{array}{ccc}-a_{2} & 1 & b_{2} g \\ 0 & c_{2} & 1-g \\ 0 & d_{2} g & -h_{2}\end{array}\right] \tilde{X}(t)+B u(t)$

Rule 2: if $\tilde{x}$ is $M_{1}$ \& $\tilde{y}$ is $N_{2}$ then $\dot{\tilde{X}}(t)=$ $\left[\begin{array}{ccc}-a_{2} & 1 & -b_{2} g \\ 0 & c_{2} & 1-g \\ 0 & d_{2} g & -h_{2}\end{array}\right] \tilde{X}(t)+B u(t)$

Rule 3: if $\tilde{x}$ is $M_{2}$ \& $\tilde{y}$ is $N_{1}$ then $\dot{\tilde{X}}(t)=$ $\left[\begin{array}{ccc}-a_{2} & 1 & b_{2} g \\ 0 & c_{2} & 1+g \\ 0 & -d_{2} g & -h_{2}\end{array}\right] \tilde{X}(t)+B u(t)$

Rule 4: if $\tilde{x}$ is $M_{2}$ \& $\tilde{y}$ is $N_{2}$ then $\dot{\tilde{X}}(t)=$ $\left[\begin{array}{ccc}-a_{2} & 1 & -b_{2} g \\ 0 & c_{2} & 1+g \\ 0 & -d_{2} g & -h_{2}\end{array}\right] \tilde{X}(t)+B u(t)$

Where

$$
\begin{aligned}
& u(t)=\left[\begin{array}{lll}
u_{1}(t) & u_{2}(t) & u_{3}(t)
\end{array}\right]^{T} \\
& B=\left[\begin{array}{lll}
1 & 0 & 0 \\
0 & 1 & 0 \\
0 & 0 & 1
\end{array}\right]
\end{aligned}
$$

Assume that $e(t)=\tilde{X}(t)-X(t)$, we obtain

$\dot{e}(t)=$

$\sum_{i=1}^{4} M_{i}(x) N_{i}(y) A_{i} X(t)-\sum_{i=1}^{4} M_{i}(\tilde{x}) N_{i}(\tilde{y})\left(A_{i} \tilde{X}(t)+\right.$ $B u(t))$ 
Rewrite the equation (34), we obtain

Rule 1: if $\tilde{x}$ is $M_{1}$ \& $\tilde{y}$ is $N_{1}$ then $\dot{e}(t)=A_{1} e(t)+$ $A_{1} X(t)+B u(t)$

Rule 2: if $\tilde{x}$ is $M_{1}$ \& $\tilde{y}$ is $N_{2}$ then $\dot{e}(t)=A_{2} e(t)+$ $A_{2} X(t)+B u(t)$

Rule 3: if $\tilde{x}$ is $M_{2}$ \& $\tilde{y}$ is $N_{1}$ then $\dot{e}(t)=A_{3} e(t)+$ $A_{3} X(t)+B u(t)$

Rule 4: if $\tilde{x}$ is $M_{2}$ \& $\tilde{y}$ is $N_{2}$ then $\dot{e}(t)=A_{4} e(t)+$

$\hat{A}_{4} X(t)+B u(t)$

Where

$A_{1}=\left[\begin{array}{ccc}-a_{2} & 1 & b_{2} g \\ 0 & c_{2} & 1-g \\ 0 & d_{2} g & -h_{2}\end{array}\right]$

$\hat{A}_{1}=\left[\begin{array}{ccc}a_{1}-a_{2} & 1 & \left(b_{2}-b_{1}\right) g \\ 0 & c_{2}-c_{1} & f_{1} g-1+g \\ 0 & \left(d_{2}-d_{1}\right) g & h_{1}-h_{2}\end{array}\right]$

$A_{2}=\left[\begin{array}{ccc}-a_{2} & 1 & -b_{2} g \\ 0 & c_{2} & 1-g \\ 0 & d_{2} g & -h_{2}\end{array}\right]$

$A_{2}=\left[\begin{array}{ccc}a_{1}-a_{2} & 1 & \left(b_{1}-b_{2}\right) g \\ 0 & c_{2}-c_{1} & f_{1} g-1+g \\ 0 & \left(d_{1}-d_{2}\right) g & h_{1}-h_{2}\end{array}\right]$

$A_{3}=\left[\begin{array}{ccc}-a_{2} & 1 & b_{2} g \\ 0 & c_{2} & 1+g \\ 0 & -d_{2} g & -h_{2}\end{array}\right]$

$\hat{A}_{3}=\left[\begin{array}{ccc}a_{1}-a_{2} & 1 & \left(b_{2}-b_{1}\right) g \\ 0 & c_{2}-c_{1} & 1+g-f_{1} g \\ 0 & \left(d_{2}-d_{1}\right) g & h_{1}-h_{2}\end{array}\right]$

$A_{4}=\left[\begin{array}{ccc}-a_{2} & 1 & -b_{2} g \\ 0 & c_{2} & 1+g \\ 0 & -d_{2} g & -h_{2}\end{array}\right]$

$\hat{A}_{4}=\left[\begin{array}{ccc}a_{1}-a_{2} & 1 & \left(b_{1}-b_{2}\right) g \\ 0 & c_{2}-c_{1} & 1+g-f_{1} g \\ 0 & \left(d_{1}-d_{2}\right) g & h_{1}-h_{2}\end{array}\right]$

According to the Active Control Method, the control signals will be obtained:

Rule 1: if $x$ is $M_{1} \& y$ is $N_{1}$ then

$\left[\begin{array}{l}u_{1}(t) \\ u_{2}(t) \\ u_{3}(t)\end{array}\right]=-A_{1} X(t)+B\left[\begin{array}{l}v_{1}(t) \\ v_{2}(t) \\ v_{3}(t)\end{array}\right] \&\left[\begin{array}{l}v_{1}(t) \\ v_{2}(t) \\ v_{3}(t)\end{array}\right]=$

$-\left[\begin{array}{ccc}-a_{2}+k_{1} & 1 & b_{2} g \\ 0 & c_{2}+k_{2} & 1-g \\ 0 & d_{2} g & -h_{2}+k_{3}\end{array}\right] e(t)$

Rule 2: if $x$ is $M_{1} \& y$ is $N_{2}$ then

$$
\begin{aligned}
& {\left[\begin{array}{l}
u_{1}(t) \\
u_{2}(t) \\
u_{3}(t)
\end{array}\right]=-A_{2} X(t)+B\left[\begin{array}{l}
v_{1}(t) \\
v_{2}(t) \\
v_{3}(t)
\end{array}\right] \&\left[\begin{array}{l}
v_{1}(t) \\
v_{2}(t) \\
v_{3}(t)
\end{array}\right]=} \\
& -\left[\begin{array}{ccc}
-a_{2}+k_{1} & 1 & -b_{2} g \\
0 & c_{2}+k_{2} & 1-g \\
0 & d_{2} g & -h_{2}+k_{3}
\end{array}\right] e(t)
\end{aligned}
$$

Rule 3: if $x$ is $M_{2}$ \& $y$ is $N_{1}$ then

$$
\begin{gathered}
{\left[\begin{array}{l}
u_{1}(t) \\
u_{2}(t) \\
u_{3}(t)
\end{array}\right]=-\hat{A}_{3} X(t)+B\left[\begin{array}{l}
v_{1}(t) \\
v_{2}(t) \\
v_{3}(t)
\end{array}\right] \&\left[\begin{array}{l}
v_{1}(t) \\
v_{2}(t) \\
v_{3}(t)
\end{array}\right]=} \\
-\left[\begin{array}{ccc}
-a_{2}+k_{1} & 1 & b_{2} g \\
0 & c_{2}+k_{2} & 1+g \\
0 & -d_{2} g & -h_{2}+k_{3}
\end{array}\right] e(t)
\end{gathered}
$$

Rule 4: if $x$ is $M_{2}$ \& $y$ is $N_{2}$ then

$$
\begin{aligned}
& {\left[\begin{array}{l}
u_{1}(t) \\
u_{2}(t) \\
u_{3}(t)
\end{array}\right]=-\dot{A}_{4} X(t)+B\left[\begin{array}{l}
v_{1}(t) \\
v_{2}(t) \\
v_{3}(t)
\end{array}\right] \&\left[\begin{array}{l}
v_{1}(t) \\
v_{2}(t) \\
v_{3}(t)
\end{array}\right]=} \\
& -\left[\begin{array}{ccc}
-a_{2}+k_{1} & 1 & -b_{2} g \\
0 & c_{2}+k_{2} & 1+g \\
0 & -d_{2} g & -h_{2}+k_{3}
\end{array}\right] e(t)
\end{aligned}
$$

Where $k_{i}, i=1,2,3$ are positive gains. We select the gains of controllers (37) $k_{1}=10, k_{2}=10, k_{3}=10$. The initial values of the the drive and response systems are $x_{1}(0)=2.2, y_{1}(0)=2.4, z_{1}(0)=3$ and $x_{2}(0)=$ $-2.2, y_{2}(0)=-2.4, z_{2}(0)=-3$ respectively. The time response of $x, y, z$ states for drive system (31) and the response system (32) shown in Figure 10. Synchronization errors $\left(e_{x}, e_{y}, e_{z}\right)$ shown in Figure 11 . The time response of the control inputs $\left(u_{1}, u_{2}, u_{3}\right)$ for the synchronization two different fuzzy chaotic systems shown in Figure 12.
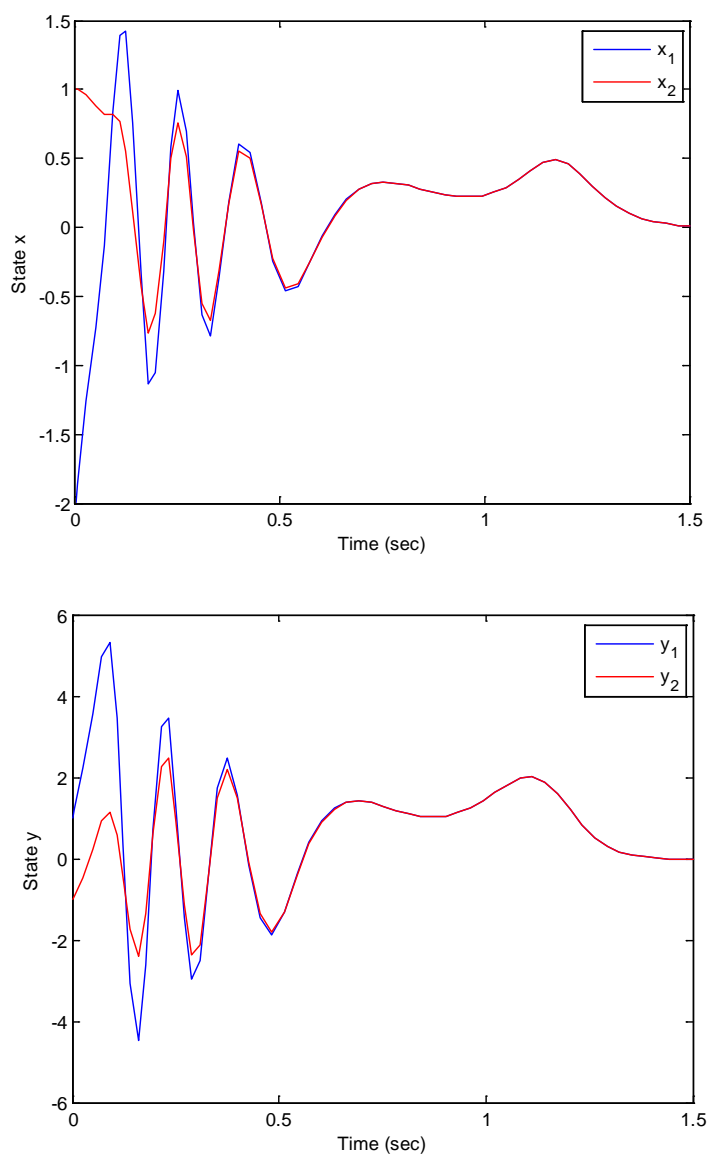


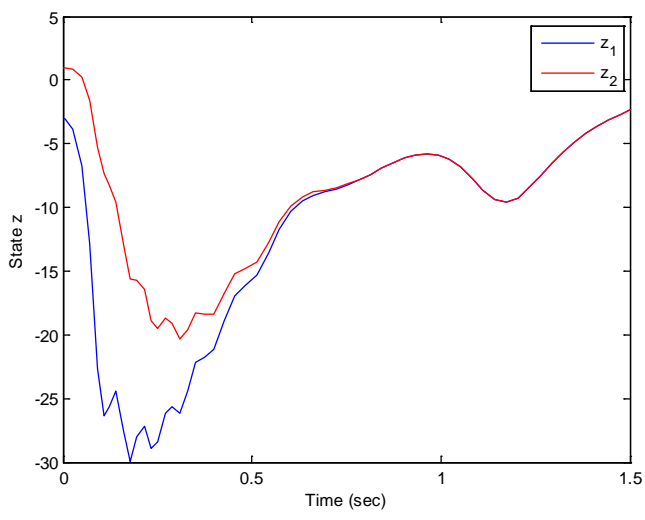

Fig 10 . The time response of signals $(x, y, z)$ for drive system (31) and response system (32).
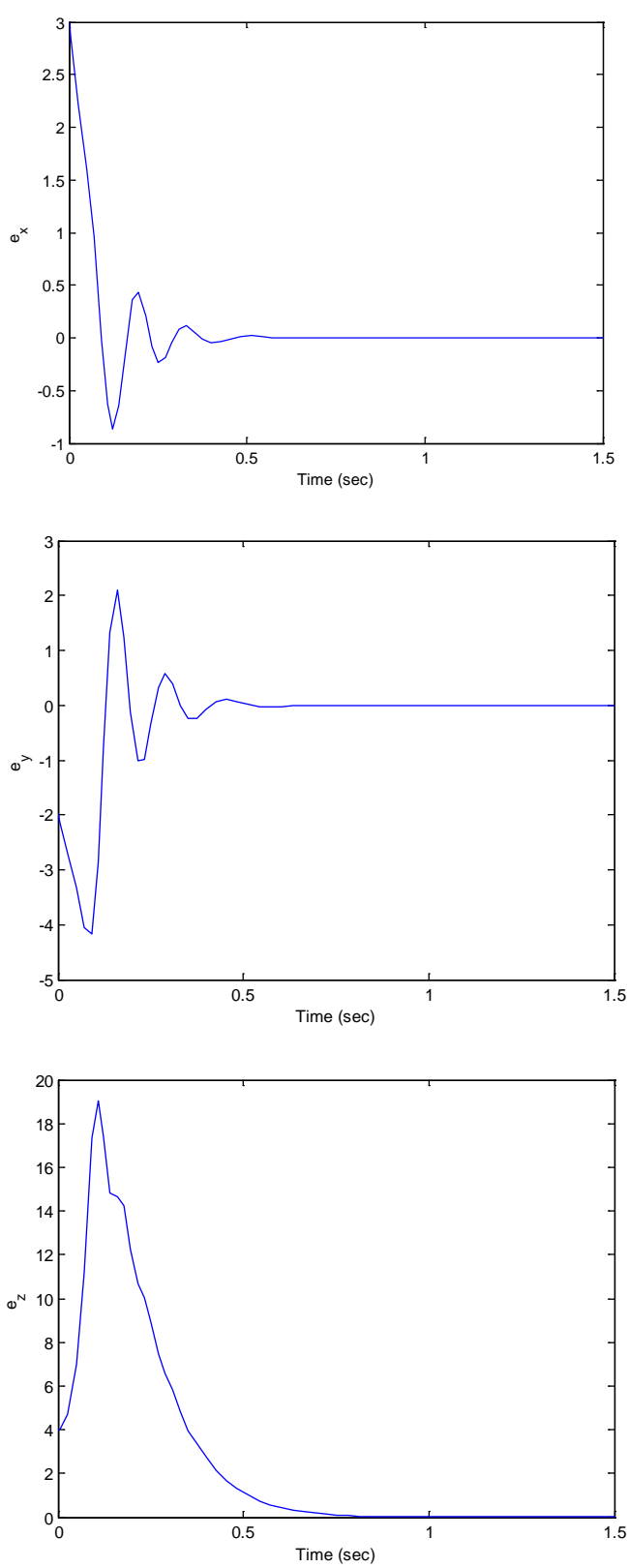

Fig 11. Synchronization errors $\left(e_{x}, e_{y}, e_{z}\right)$ in drive system (31) and response system (32).
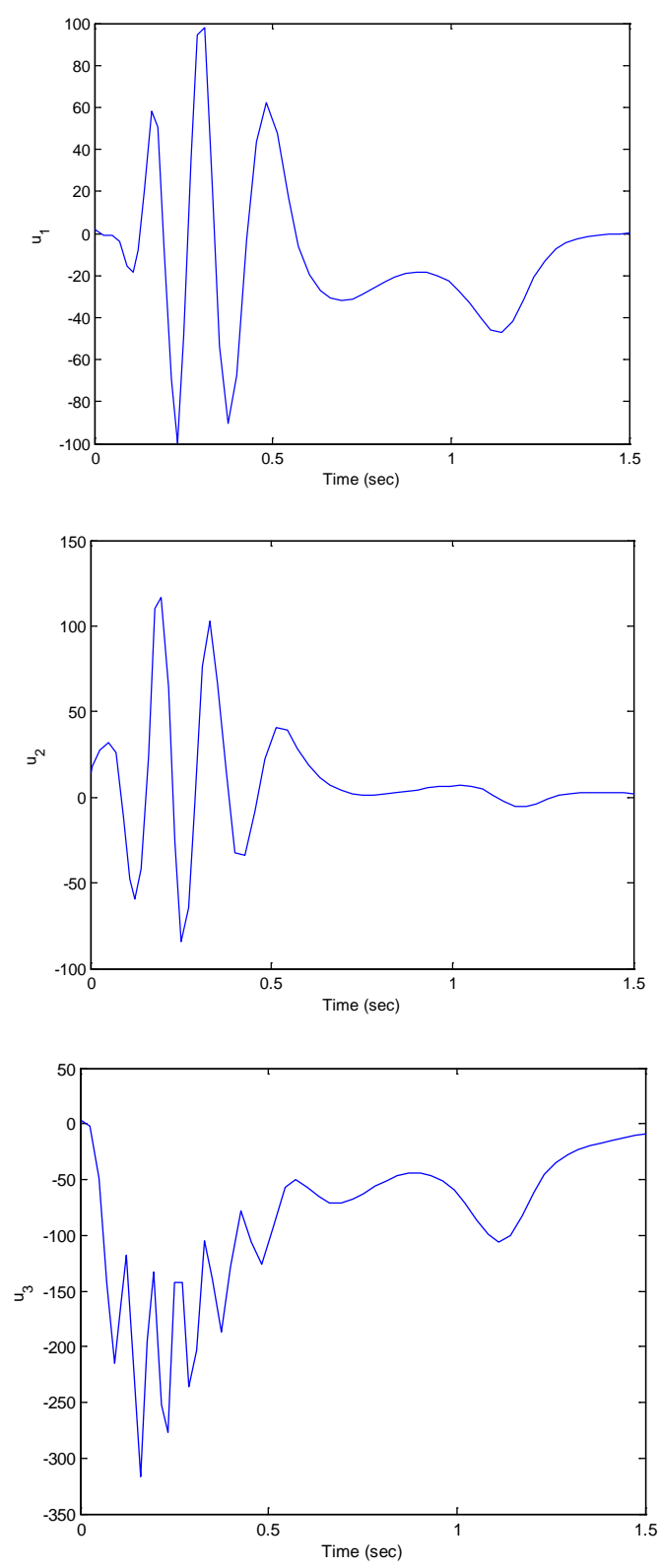

Fig 12. The time response of the control inputs $\left(u_{1}, u_{2}, u_{3}\right)$ for drive system (31) and response system (32).

Substituting equation (37) into equation (32) yields.

Rule 1: if $\tilde{x}$ is $M_{1}$ \& $\tilde{y}$ is $N_{1}$ then $\dot{e}(t)=A_{1} e(t)$

Rule 2: if $\tilde{x}$ is $M_{1}$ \& $\tilde{y}$ is $N_{2}$ then $\dot{e}(t)=A_{2} e(t)$

Rule 3: if $\tilde{x}$ is $M_{2}$ \& $\tilde{y}$ is $N_{1}$ then $\dot{e}(t)=A_{3} e(t)$

Rule 4: if $\tilde{x}$ is $M_{2}$ \& $\tilde{y}$ is $N_{2}$ then $\dot{e}(t)=A_{4} e(t)$

Where

$$
A_{1}=A_{2}=A_{3}=A_{4}=\left[\begin{array}{ccc}
-k_{1} & 0 & 0 \\
0 & -k_{2} & 0 \\
0 & 0 & -k_{3}
\end{array}\right]
$$

We select $Q=I_{3 \times 3}$ and the matrix $P$ will be obtained:

$$
A_{i}^{T} P+P A_{i}=-Q, \quad i=1,2,3,4
$$




$$
P=\frac{1}{20} I_{3 \times 3}
$$

\section{CONCLUSION}

In this paper, a fuzzy model-based designs for new 3D chaotic stability and synchronization have been proposed. Based on the fuzzy chaotic models, simpler fuzzy controllers have been designed for stabilizing and synchronizing new chaotic systems via generalized backstepping method. Then the synchronization of two different chaotic system are achieved by solving active control. Finally, numerical simulation was given to verify the effectiveness of the proposed controllers.

\section{REFERENCES}

[1] L. M. P. a. T. L. Carroll, Synchronization in chaotic systems, Phys. Rev. Lett., 64,, pp. 821-824, 1990.

[2] C.-W. Park, C.-H. Lee, M. Park, Inform. Sci. 147 (2002) 245.

[3] L. Chen, G. Chen, Y.-W. Lee, Inform. Sci. 121 (1999) 27.

[4] K.-Y. Lian, C.-S. Chiu, T.-S. Chiang, P. Liu, IEEE Trans. Fuzzy Systems 9 (4) (2001) 539.

[5] Yan-Wu Wang, Zhi-Hong Guan, Hua O. Wang, LMIbased fuzzy stability and synchronization of Chen's system, Physics Letters A 320 (2003) 154-159.

[6] Hongbin Zhang, Xiaofeng Liao, Juebang Yu, Fuzzy modeling and synchronization of hyperchaotic systems, Chaos, Solitons and Fractals 26 (2005) 835-843.

[7] Mojtaba Ahmadieh Khanesar, Mohammad Teshnehlab, Okyay Kaynak, Control and synchronization of chaotic systems using a novel indirect model reference fuzzy controller, Soft Comput, DOI 10.1007/s00500-012-0810-z.

[8] Xiulan ZHANG, Fengyun ZHANG, Synchronizing Uncertain Chaotic Systems by Using Adaptive Fuzzy Chattering Free Sliding Mode Control, Journal of Computational Information Systems 8: 24 (2012) 1050910515.

[9] Yonghui Sun, Zhinong Wei, Guoqiang Sun, Ping Ju, and Shuaidong Huang, Robust Stabilization and Synchronization of Nonlinear Energy Resource System via Fuzzy Control Approach, International Journal of Fuzzy Systems, Vol. 14, No. 2, June 2012.

[10] Tsung-Chih Lin and Kang-Wei Hsu, A NEW ADAPTIVE H1 CONTROL FOR CHAOS SYNCHRONIZATION BETWEEN TWO DIFFERENT CHAOTIC SYSTEMS, International Journal of Innovative Computing, Information and Control, Volume 8, Number 1(B), January 2012, pp. 701-714.

[11] Lin-jun TANG, DongLI, Han-xing WANG, Lag synchronization for fuzzy chaotic system based on fuzzy observer, Appl. Math. Mech. -Engl. Ed. 30(6), 803-810 (2009).

[12] Yaghoub Heidari, Soheil Salehi Alashti, Rouhollah Maghsoudi, Chaos Synchronization of two Uncertain Chaotic System Using Genetic Based Fuzzy Adaptive PID Controller, The Journal of Mathematics and Computer Science Vol .1 No.4 (2010) 273-286.

[13] Davood Babaei Pourkargar, Mohammad Shahrokhi, Optimal Fuzzy Synchronization of Generalized Lorenz Chaotic Systems, The Journal of Mathematics and Computer Science Vol .2 No.1 (2011) 27-36.

[14] Alireza Sahab, Masoud Taleb Ziabari, Intelligent Controller for Synchronization New Three Dimensional
Chaotic System, I.J. Modern Education and Computer Science, 2014, 7, 40-46.

[15] Masoud Taleb Ziabari, Ali Reza Sahab, Control and Synchronization of Hyperchaotic System based on SDRE Method, I.J. Information Engineering and Electronic Business, 2014, 3, 44-50.

[16] T. Takagi, M. Sugeno, IEEE Trans. Systems Man Cybernet. 15 (1) (1985) 116.

[17] K. Tanaka, H.O. Wang, Fuzzy Control Systems Design and Analysis: A Linear Matrix Inequality Approach, Wiley, New York, 2001.

[18] Sara Dadras, Hamid Reza Momeni, A novel threedimensional autonomous chaotic system generating two, three and four-scroll attractors, Physics Letters A 373 (2009) 3637-3642.

[19] Ali Reza Sahab and Mohammad Haddad Zarif, Improve Backstepping Method to GBM, World Applied Sciences Journal 6 (10): 1399-1403, 2009.

[20] Ali Reza Sahab, Masoud Taleb Ziabari and Mohammad Reza Modabbernia, A novel fractional-order hyperchaotic system with a quadratic exponential nonlinear term and its synchronization, Advances in Difference Equations 2012, 2012:194.

[21] Chunlai Li, Hongmin Li, Yaonan Tong, Analysis of a novel three-dimensional chaotic system, Optik - Int. J. Light Electron Opt. (2012), http://dx.doi.org/10.1016/j.ijleo.2012.04.005.

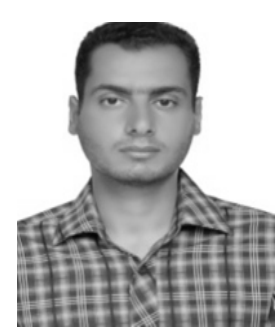

Masoud Taleb Ziabari. Received the BSc in computer hardware from Islamic Azad University, Yazd, Iran in 2005. He received the MSc degree in mechatronic in Islamic Azad University Qazvin Branch, Qazvin, Iran. His research interests include nonlinear control and chaotic systems.

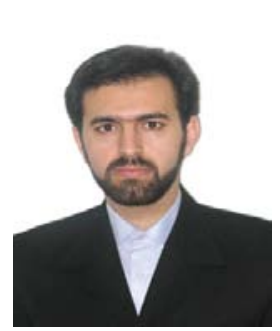

Ali Moarefianpour. Received the B.S. degree in control system engineering from Isfahan University of Technology, Isfahan, Iran in 2000 and M.S. and Ph.D degrees in control system engineering from TarbiatModaressUniversity, Tehran, Iran in 2002 and 2008 respectively.Currently, he is a Lecturer in Islamic Azad University, Science and Research Branch. His research interests include fuzzy control theory, intelligent control and Process automation.

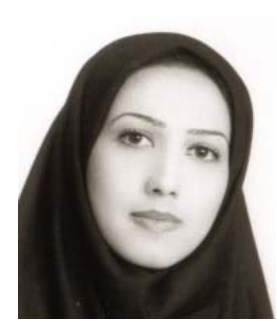

Marjan Morvarid. Received the B.S in computer hardware from Islamic Azad University, Qazvin, Iran in 2005 and M.S in computer systems architecture in Iran University of Science and Technology, Tehran, Iran in 2009. She is faculty member of computer engineering Group, AhrarInstitue of Technology and Higher Education, Rasht, Iran. Her research interests includes network on chip. 